Original paper

\title{
The association between obstructive sleep apnea and non-alcoholic steatohepatitis: a retrospective nationwide inpatient sample analysis
}

\author{
Anas Renno, Yousef Abdel-Aziz, Yaseen Alastal, Sadik Khuder, Syed Hasan, Ragheb Assaly, Ali Nawras \\ University of Toledo Medical Center, United States
}

\begin{abstract}
Aim of the study: The primary purpose of this study was to assess the association of obstructive sleep apnea (OSA) and non-alcoholic steatohepatitis (NASH) from a large national inpatient sample database.

Material and methods: We conducted a retrospective analysis using the Healthcare Cost and Utilization Project-Nationwide Inpatient Sample. OSA and NASH patients were identified using the ICD-10-CM code G47.33 and K75.81. Non-NASH patients (control) were randomly selected and matched by age and gender to each NASH patient in a $4: 1$ ratio. Weighted logistic regression models were used to calculate the association between OSA and NASH in addition to different comorbidities.

Results: A total of 54,169 participants were included in our analysis; 10,740 cases of NASH were matched to 43,429 controls (non-NASH). NASH was significantly higher in the white population ( $82.12 \%$ vs. $76.64 \%$, $p<0.001)$. The prevalence of OSA among NASH patients was significantly higher compared to the control group (15.8\% vs. $8.9 \%$, adjusted OR: $1.34,95 \% \mathrm{Cl}: 1.14-1.56, p=0.0003)$. The prevalence of celiac disease and Crohn's disease was significantly higher in patients with NASH $(0.7 \%$ vs. $0.2 \%, p<0.0002$ and $1.28 \%$ vs. $0.76 \%, p<0.0001)$. Multiple comorbidities were significantly elevated in the NASH group compared to the non-NASH group, including diabetes mellitus (DM; $36 \%$ vs. $17.6 \%, p<0.0001$ ), obesity ( $36.4 \%$ vs. $18.2 \%$, $p<0.0001)$ and metabolic syndrome $(0.86 \%$ vs. $0.06 \%, p<0.0001)$. The mortality rate was significantly higher in the NASH group ( $3.8 \%$ vs. $2 \%, p<0.0001)$.
\end{abstract}

Conclusions: This is the first study using the ICD-10-CM code with a specific search code for NASH. Our large population database results emphasize that there is a significant association between OSA and NASH.

Key words: metabolic syndrome, obstructive sleep apnea, mortality, non-alcoholic steatohepatitis.

Address for correspondence:

Dr. Anas Renno, University of Toledo Medical Center, United States, e-mail: anas.renno@utoledo.edu

\section{Introduction}

Non-alcoholic steatohepatitis (NASH) is a clinical condition that belongs to the spectrum of non-alcoholic fatty liver disease (NAFLD) [1]. Histopathologically, it is characterized by inflammation, hepatocyte swelling, and steatosis [2]. Moreover, in $15 \%$ to $20 \%$ of patients, NASH may lead to hepatic cirrhosis $[3,4]$. Another serious clinical condition is obstructive sleep apnea (OSA), characterized by upper airway obstruction (causing intermittent hypoxia) and interrupted sleep [5]. Both conditions have been associated togeth- er as a cause/result or potential co-occurring complications of obesity $[6,7]$.

One of the prevalent theories for the development of NASH is the two-hit hypothesis. This theory indicates that benign hepatic steatosis may be the first hit and then another precipitating factor (second hit) may start the pathogenesis [8]. The involvement of OSA as a second hit in NASH development is supported by both experimental and epidemiological evidence. An experiment by Zamora-Valdés et al. in mice showed that exposure to a high-fat diet along with chronic intermittent hypoxia was associated with lobular in- 
flammation and fibrosis, as well as significant increases in the hepatic levels of pro-inflammatory cytokines (interleukin $1 \beta$ and 6 and tumor necrosis factor $\alpha$ ), as well as collagen-1 $\alpha$ mRNA. Other in vivo experiments showed concordant results [9]. Oxidative stress and the release of hypoxia-inducible factor- 1 are hypothesized to be the main players in this association $[10,11]$.

Simultaneously, epidemiological studies have shown a higher prevalence of NASH in OSA patients, as well as a higher prevalence of OSA in NASH patients. However, the evidence remains largely inconclusive, i.e. some studies have reported significant elevations of serum liver enzymes in OSA patients [12-14], while other studies failed to record such an observation [15-17]. This heterogeneity is probably explained by the small sample size of the existing studies and the variety of used definitions and diagnostic criteria for NASH and OSA. Therefore, a large-scale study with a well-defined definition of both conditions may provide higher-quality evidence about this association.

The primary purpose of this study was to assess the association between NASH and OSA using data from a large national inpatient sample database.

\section{Material and methods}

\section{Study design}

This retrospective cross-sectional analysis was conducted using the Healthcare Cost and Utilization Project's (HCUP) Nationwide Inpatient Sample (NIS) data from the year 2016. HCUP data are sponsored by the Agency for Healthcare Research and Quality (AHRQ) and are specifically designed to determine and identify patterns in hospital utilization and costs across the United States (https://www.hcup-us.ahrq.gov/nisoverview.jsp; accessed on August 3, 2019). This study was deemed exempt by the Institutional Review Board of the University of Toledo Medical Center, as the HCUP is a publicly available database that contains de-identified patient information.

\section{Participants}

Our study included individuals who were at least 18 years of age and had an ICD-10 diagnosis code for NASH. The NIS database allows up to 30 diagnoses per patient. Based on the International Classification of Diseases, Tenth Revision (ICD-10) diagnosis codes, we identified the target group as the patients admitted to the hospital with a diagnosis of NASH in any of the 30 possible diagnoses. We generated a matched casecontrol where the case group (patients NASH) was matched with the control group (patients without NASH) on the basis of age and sex. Four controls were matched to each NASH patient $(10,740 \mathrm{NASH}$ and 43,429 controls).

\section{Statistical analysis}

Statistical analyses were conducted using SAS version 9.2 (SAS Institute, Cary, NC). Frequency distributions between categorical variables were assessed using the $\chi^{2}$ test. An odds ratio with $95 \%$ confidence interval was calculated for each variable. Logistic regression models were used to compare patients with and without NASH for different comorbidities. The survey logistic procedures were used in this analysis to include the weight variable provided in the database. The presence of NASH was used as the dependent variable in these models. Each model was weighted and controlled for the comorbid condition as well as race and urban/rural location. A $p$-value of $<0.05$ was considered statistically significant.

\section{Results}

\section{Baseline characteristics of enrolled participants}

Following our matching approach, the age of both $\mathrm{NASH}$ and control groups was comparable $(p=0.96)$ and females represented $61.3 \%$ of each group. However, we noted significant differences between the groups in terms of race; White participants represented a significantly larger portion of the NASH group, compared to the matched controls $(82.1 \%$ vs. $76.6 \%, p<0.0001)$. No significant difference was recorded between the two groups in terms of cigarette smoking $(p=0.15)$. Table 1 illustrates the details of participants' baseline characteristics.

\section{Association between NASH and OSA}

The prevalence of OSA was significantly higher in the NASH group (OR $=1.34,95 \%$ CI: $1.14,1.56$, $p=0.0003)$ than the control group.

\section{Association between NASH and other comorbidities}

In comparison to age and sex-matched controls, the NASH group displayed higher frequency of being diagnosed with metabolic conditions as obesity $(\mathrm{OR}=2.23,95 \% \mathrm{CI}: 1.99-2.50, p<0.0001), \mathrm{DM}$ $(\mathrm{OR}=2.55,95 \%$ CI: 2.29-2.85, $p<0.0001)$, and metabolic syndrome $(\mathrm{OR}=11.36,95 \% \mathrm{CI}$ : 3.61-35.71, 
$p<0.0001)$. Similarly, we recorded a higher risk of ascites $(\mathrm{OR}=9.43$, 95\% CI: 6.85-13.16, $p<0.0001)$, esophageal varices $(\mathrm{OR}=7.04,95 \% \mathrm{CI}: 4.39-11.24, p<0.0001)$, Crohn's (OR $=1.3,95 \%$ CI: $1.18-2.86, p=0.007)$ and celiac diseases $(\mathrm{OR}=3.86,95 \% \mathrm{CI}: 1.91-7.81, p=0.0002)$ in the NASH group than the control group.

On the other hand, no significant differences were recorded between the NASH and control groups in terms of the frequency of pulmonary hypertension $(\mathrm{OR}=1.21,95 \% \mathrm{CI}: 0.96-1.52, p=0.1)$, ulcerative colitis ( $\mathrm{OR}=1.38,95 \% \mathrm{CI}$ : 0.65-2.90, $p=0.39)$, and hemochromatosis $(\mathrm{OR}=2.35,95 \%$ CI: $0.34-16.39, p=0.38)$. Table 2 summarizes the details of comorbidities in both groups.

\section{Mortality rate in NASH patients and controls}

The risk of in-hospital mortality was significantly higher in the NASH group (OR $=1.34,95 \%$ CI: 1.22 $1.48, p<0.0001$ ) than in matched controls (Fig. 1).

\section{Discussion}

This retrospective nationwide study investigated the association between NASH and different medical
Table 1. Distribution of non-alcoholic steatohepatitis (NASH) and non-NASH in relation to demographic variables

\begin{tabular}{|c|c|c|c|}
\hline Variable & $\begin{array}{c}\text { NASH } \\
n=10,950\end{array}$ & $\begin{array}{c}\text { Controls } \\
n=43,810\end{array}$ & $p$ \\
\hline Gender, female (\%) & 61.28 & 61.28 & - \\
\hline Age (mean $\pm S D$ ) & $61.21 \pm 13.92$ & $61.23 \pm 14.30$ & 0.96 \\
\hline \multicolumn{4}{|l|}{ Race $(\%)$} \\
\hline White & 82.12 & 76.61 & \multirow{6}{*}{$<0.0001^{*}$} \\
\hline Black & 4.52 & 12.07 & \\
\hline Hispanic & 8.52 & 6.20 & \\
\hline Asian or Pacific Islander & 1.26 & 2.15 & \\
\hline Native American & 0.19 & 0.17 & \\
\hline Other & 3.39 & 2.80 & \\
\hline Cigarette smoking & 1.1 & 2.33 & 0.15 \\
\hline
\end{tabular}

conditions, including, but not limited to, OSA. We found a significant association between NASH and OSA in a sample of 54,169 participants. Moreover, we detected a significant association between NASH and obesity, DM, and metabolic syndrome, hinting at the possible involvement of these conditions in the pathogenesis of NASH. However, there was no significant

Table 2. Distribution of selected co-morbidities between patients with and without non-alcoholic steatohepatitis (NASH)

\begin{tabular}{|c|c|c|c|c|}
\hline Co-morbidities & $\begin{array}{l}\text { NASH, } n(\%) \\
(n=10,950)\end{array}$ & $\begin{array}{c}\text { Control, } n(\%) \\
(n=43,810)\end{array}$ & $\begin{array}{c}\text { OR } \\
95 \% \mathrm{Cl}\end{array}$ & $p$ \\
\hline Obstructive sleep apnea (OSA) & $1740(15.89)$ & $3890(8.88)$ & $\begin{array}{c}1.34 \\
1.14-1.56\end{array}$ & $0.0003^{*}$ \\
\hline Diabetes mellitus & 3950 (36.1) & $7740(17.67)$ & $\begin{array}{c}2.55 \\
2.29-2.85\end{array}$ & $<0.0001^{*}$ \\
\hline Obesity & $3990(36.44)$ & $8000(18.26)$ & $\begin{array}{c}2.23 \\
1.99-2.5\end{array}$ & $<0.0001^{*}$ \\
\hline Metabolic syndrome & $95(0.87)$ & $25(0.06)$ & $\begin{array}{c}11.36 \\
3.61-35.71\end{array}$ & $<0.0001^{*}$ \\
\hline Pulmonary hypertension & $600(5.48)$ & $1765(4.03)$ & $\begin{array}{c}1.21 \\
0.96-1.52\end{array}$ & 0.10 \\
\hline Ascites & $2685(24.52)$ & $440(1.00)$ & $\begin{array}{c}9.43 \\
6.85-13.16\end{array}$ & $<0.0001^{*}$ \\
\hline Esophageal varices & $1995(18.22)$ & $255(0.58)$ & $\begin{array}{c}7.04 \\
4.39-11.24\end{array}$ & $<0.0001^{*}$ \\
\hline Ulcerative colitis & $90(0.82)$ & $260(0.59)$ & $\begin{array}{c}1.38 \\
0.65-2.90\end{array}$ & 0.39 \\
\hline Crohn's disease & $140(1.28)$ & $335(0.76)$ & $\begin{array}{c}1.83 \\
1.18-2.86\end{array}$ & $0.007^{*}$ \\
\hline Hemochromatosis & $15(0.14)$ & $30(0.07)$ & $\begin{array}{c}2.35 \\
0.34-16.39\end{array}$ & 0.38 \\
\hline Celiac disease & $75(0.68)$ & $95(0.21)$ & $\begin{array}{c}3.86 \\
1.91-7.81\end{array}$ & $0.0002^{*}$ \\
\hline
\end{tabular}

$O R$ - odds ratio, $\mathrm{Cl}$ - confidence interval, *indicates significance at $p<0.05$ 


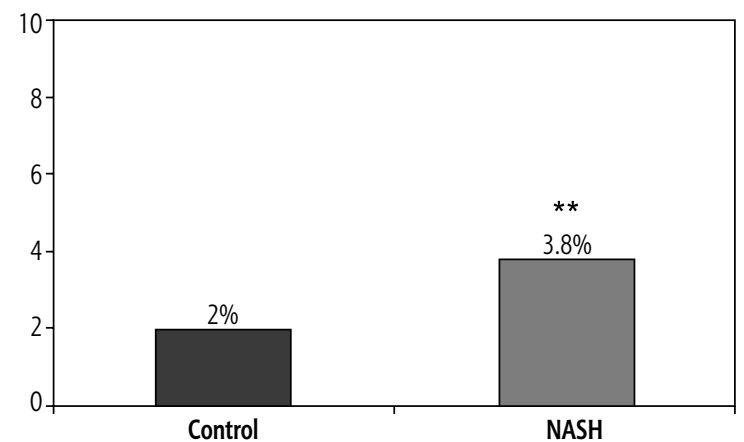

Fig. 1. Risk of in-hospital mortality in NASH and control groups. Columns represent percentages of mortality in both groups. ${ }^{*}$ indicates significance at $p<0.001$

association between NASH and pulmonary hypertension, ulcerative colitis, or hemochromatosis.

The literature on the association between NASH and OSA has been controversial. Various studies have reported conflicting results between supporting [12-14] and negating it [15-17]. However, a meta-analysis of 11 studies identified a significant association between OSA and elevated liver enzymes, as well as a 2.6-fold higher risk of developing liver fibrosis among those who had NAFLD [18]. However, the majority of these studies focused on obese individuals, who may not represent the entire population. Therefore, a large, nationwide study was required. The current study provides further evidence on the significant association between NASH and OSA.

Several mechanisms were postulated to explain this association. Oxidative stress remains the predominant hypothesis. This occurs through repetitive cycles of hypoxia/reoxygenation, which disturb mitochondrial respiration, as well as bouts of catecholamine release, inducing metabolic changes $[10,11]$. Moreover, hypoxia stimulates fibrosis and angiogenesis by enhancing the expression of hypoxia-inducible factor-1a, vascular endothelial growth factor, angiotensin-I-converting enzyme, and transforming growth factor $\beta 1$ $[19,20]$. In addition, hypoxia is an established risk factor for inflammation [9].

In addition, this study documented a significant association between NASH and other comorbidities such as obesity, DM, and metabolic syndrome. Based on previous evidence, obesity, OSA and NASH seem to form a triad $[16,21]$ in which obesity leads to hepatic steatosis, OSA leads to hypoxia and oxidative stress, and both predispose to the development of NASH [22, 23]. However, the role of each condition in this triad requires further elucidation. Diabetes also commonly co-occurs with NASH. OSA was suggested as a contributor to this association where it augments insulin resistance, hence inducing hepatic steatosis. Previous epidemiological studies showed a significant associa- tion between NASH and type 2 DM $[24,25]$. The association between NASH and hypertension, obesity, and DM draws the full picture of metabolic syndrome.

Interestingly, our study showed a significant association between NASH and Crohn's disease. Although this association was reported previously, the majority of relevant publications were case reports/series [28]. To our knowledge, this is the largest study to report on this association. Ulcerative colitis has been more frequently reported in association with NAFLD [28]; however, the current study did not provide any evidence of such an association. Further prospective, large-scale studies are required to verify the existence of such an association. Another interesting association in our analysis was between $\mathrm{NASH}$ and celiac disease. This association has been highlighted before $[29,30]$, and disturbance of the gut-liver axis and increased permeability to intraluminal antigens have been suggested as potential mechanisms [31].

To our knowledge, this is the largest study on the association between OSA and NASH. However, it has some limitations. First, we could not assess the effects of OSA on the severity of NASH because data on the severity of NASH were not reported in our database. The nationwide inpatient data cannot specify the method of diagnosis as to whether it is biopsy proven or based on imaging studies. We performed age and sex matching; however, we could not exclude the presence of other confounders such as obesity and lifestyle habits. Moreover, the results here merely prove the association without defining its direction, i.e. which condition causes the other or whether both conditions are just consequences of obesity. Prospective studies may provide better results in this regard. The outcomes in these studies should not be simply the diagnosis of NASH, but should include the histological characteristics of NASH such as hepatocyte ballooning. Moreover, therapeutic interventions for OSA in NASH patients such as continuous positive airway pressure (CPAP) have produced controversial results $[32,33]$ and need better assessment in well-powered randomized controlled trials.

In conclusion, this is the first study using the ICD10-CM code with a specific search code for NASH. Our large population database results emphasize that there is a significant association between OSA and NASH. This association should prompt physicians to evaluate for the presence of OSA in NASH patients. Moreover, it adds further evidence to the previously reported association between NASH and obesity, DM, and metabolic syndrome.

\section{Disclosure}

The authors declare no conflict of interest. 


\section{References}

1. Vernon G, Baranova A, Younossi Z. Systematic review: the epidemiology and natural history of non-alcoholic fatty liver disease and non-alcoholic steatohepatitis in adults. Aliment Pharmacol Ther 2011; 34: 274-285.

2. Rinella ME. Nonalcoholic fatty liver disease: a systematic review. JAMA 2015; 313: 2263-2273.

3. Ekstedt M, Franzén LE, Mathiesen UL, et al. Long-term followup of patients with NAFLD and elevated liver enzymes. Hepatology 2006; 44: 865-873.

4. Adams LA, Sanderson S, Lindor KD, et al. The histological course of nonalcoholic fatty liver disease: a longitudinal study of 103 patients with sequential liver biopsies. J Hepatol 2005; 42: 132-138.

5. Senaratna CV, Perret JL, Lodge CJ, et al. Prevalence of obstructive sleep apnea in the general population: a systematic review. Sleep Med Rev 2017; 34: 70-81.

6. Daltro C, Cotrim HP, Alves E, et al. Nonalcoholic fatty liver disease associated with obstructive sleep apnea: just a coincidence? Obes Surg 2010; 20: 1536-1543.

7. Jouët P, Sabaté JM, Maillard D, et al. Relationship between obstructive sleep apnea and liver abnormalities in morbidly obese patients: a prospective study. Obes Surg 2007; 17: 478-485.

8. James O, Day C. Non-alcoholic steatohepatitis: another disease of affluence. The Lancet 1999; 353: 1634-1636.

9. Zamora-Valdés D, Méndez-Sánchez N. Experimental evidence of obstructive sleep apnea syndrome as a second hit accomplice in nonalcoholic steatohepatitis pathogenesis. Ann Hepatol 2007; 6: 281-283.

10. Musso G, Olivetti C, Cassader M, et al. Obstructive sleep apnea-hypopnea syndrome and nonalcoholic fatty liver disease: emerging evidence and mechanisms. Semin Liver Dis 2012; 32: 49-64.

11. Carabelli J, Burgueño AL, Soledad Rosselli M, et al. High fat diet-induced liver steatosis promotes an increase in liver mitochondrial biogenesis in response to hypoxia. J Cell Mol Med 2011; 15: 1329-1338.

12. Byrne TJ, Parish JM, Somers V, et al. Evidence for liver injury in the setting of obstructive sleep apnea. Ann Hepatol 2012; 11: 228-231.

13. Kheirandish-Gozal L, Sans Capdevila O, Kheirandish E, et al. Elevated serum aminotransferase levels in children at risk for obstructive sleep apnea. Chest 2008; 133: 92-99.

14. Kallwitz ER, Herdegen J, Madura J, et al. Liver enzymes and histology in obese patients with obstructive sleep apnea. J Clin Gastroenterol 2007; 41: 918-921.

15. Acartürk G, Unlü M, Yüksel S, et al. Obstructive sleep apnoea, glucose tolerance and liver steatosis in obese women. J Int Med Res 2007; 35: 458-466.

16. Polotsky VY, Patil SP, Savransky V, et al. Obstructive sleep apnea, insulin resistance, and steatohepatitis in severe obesity. Am J Respir Crit Care Med 2009; 179: 228-234.

17. Tatsumi K, Saibara T. Effects of obstructive sleep apnea syndrome on hepatic steatosis and nonalcoholic steatohepatitis. Hepatol Res 2005; 33: 100-104.

18. Sookoian S, Pirola CJ. Obstructive sleep apnea is associated with fatty liver and abnormal liver enzymes: a meta-analysis. Obes Surg 2013; 23: 1815-1825.

19. Rosmorduc O, Housset C. Hypoxia: a link between fibrogenesis, angiogenesis, and carcinogenesis in liver disease. Semin Liver Dis 2010; 30: 258-270.
20. Sookoian S, Fernández Gianotti T, Soledad Rosselli M, et al Liver transcriptional profile of atherosclerosis-related genes in human nonalcoholic fatty liver disease. Atherosclerosis 2011; 218: 378-385.

21. Nobili V, Cutrera R, Liccardo D, et al. Obstructive sleep apnea syndrome affects liver histology and inflammatory cell activation in pediatric nonalcoholic fatty liver disease, regardless of obesity/insulin resistance. Am J Respir Crit Care Med 2014; 189: 66-76

22. Yu J, Shen J, Sun TT, et al. Obesity, insulin resistance, NASH and hepatocellular carcinoma. Semin Cancer Biol 2013; 23: 483-491.

23. Marchesini G, Marzocchi R. Metabolic syndrome and NASH. Clin Liver Dis 2007; 11: 105-117.

24. Bugianesi E, Vanni E, Marchesini G. NASH and the risk of cirrhosis and hepatocellular carcinoma in type 2 diabetes. Curr Diab Rep 2007; 7: 175-180.

25. Youssef W, McCullough A. Diabetes mellitus, obesity, and hepatic steatosis. Semin Gastrointest Dis 2002; 13: 17-30.

26. Tomizawa M, Kawanabe Y, Shinozaki F, et al. Triglyceride is strongly associated with nonalcoholic fatty liver disease among markers of hyperlipidemia and diabetes. Biomed Rep 2014; 2: 633-636.

27. Hyogo H, Ikegami T, Tokushige K, et al. Efficacy of pitavastatin for the treatment of non-alcoholic steatohepatitis with dyslipidemia: An open-label, pilot study. Hepatol Res 2011; 41: 10571065.

28. McGowan CE, Jones P, Long MD, et al. Changing shape of disease: nonalcoholic fatty liver disease in Crohn's disease - a case series and review of the literature. Inflamm Bowel Dis 2011; 18: 49-54.

29. Bardella M, Valenti L, Pagliari C, et al. Searching for coeliac disease in patients with non-alcoholic fatty liver disease. Dig Liver Dis 2004; 36: 333-336.

30. Reilly NR, Lebwohl B, Hultcrantz R, et al. Increased risk of non-alcoholic fatty liver disease after diagnosis of celiac disease. J Hepatol 2015; 62: 1405-1411.

31. Abenavoli L, Milic N, De Lorenzo A, et al. A pathogenetic link between non-alcoholic fatty liver disease and celiac disease. Endocrine 2013; 43: 65-67.

32. Shpirer I, Copel L, Broide E, et al. Continuous positive airway pressure improves sleep apnea associated fatty liver. Lung 2010; 188: 301-307.

33. Sivam S, Phillips CL, Trenell MI, et al. Effects of 8 weeks of continuous positive airway pressure on abdominal adiposity in obstructive sleep apnoea. Eur Respir J 2012; 40: 913-918. 